\title{
Patent ductus arteriosus - clinical profile and outcome after treatment in a tertiary care hospital
}

\author{
Dinakara $\mathbf{P}^{1}$, Bharat Reddy $D^{2}$, Rajeshwari $\mathbf{S}^{3}$, Utkarsh $\mathbf{J}^{4}$ \\ ${ }^{1}$ Dr Dinakara Prithviraj, Associate Professor, ${ }^{2}$ Dr. Bharath Reddy, Assistant Professor, ${ }^{3}$ Dr. Rajeshwari S, Junior \\ Resident, ${ }^{4}$ Dr. Utkarsh Jain, Junior Resident, all authors are affiliated with Department of Pediatrics, Vydehi Institute of \\ Medical Sciences and Research Institute, Bengaluru, Karnataka, India.
}

Address of Correspondence: Dr Dinakara Prithviraj, Associate Professor, Department of Pediatrics, Vydehi Institute of Medical Sciences, Bangalore. E-mail: drdinakar.nishanth@gmail.com

\begin{abstract}
Background: Patent ductus arteriosus (PDA) is the commonest congenital heart disease seen in neonatal ICU. Methods: A prospective observational study was conducted in the neonatal intensive care unit of Vydehi institute of medical sciences between January 2013 to December 2016. Neonates suspected to have congenital heart disease in the NICU on the basis of history clinical examination, pulse oximetry, chest $\mathrm{X}$ ray were included in the study and confirmed by $2 \mathrm{D}$ echocardiography and colour Doppler study. PDA if found hemodynamically significant treated with a three-day course of Ibubrufen and data analyzed. Results: A total of 2658 cases were admitted in the NICU during the study period of which 1974 cases were screened for CHDs. Out of 1974 babies, Echo done showed 65 babies had PDA. Out of 65 cases with PDA 32 were hemodynamically significant. About 25 out of 32 cases had responded to treatment with Ibubrufen. Ductus were closed in 5 out of 9 large PDAs. About $48 \%$ of the PDAs were closed by $5^{\text {th }}$ day of starting the treatment. Conclusion: The study shows PDA is a commonest Acyanotic CHD in NICU and early administration of the drug is helpful in closure, avoiding surgical correction later and also mortality.
\end{abstract}

Keywords: Patent ductus arteriosus, Screening, Echocardiography

\section{Introduction}

Patent ductus arteriosus (PDA) is the most common CHD in the Preterms and one of the commonest CHD seen in neonatal ICU [1]. The ductus arteriosus in normal term infants constricts rapidly after birth, resulting in ductal intimal hypoxia, remodeling and permanent closure. In most term infants, this process is well established within 72 hours of birth. Most well preterm infants $>29$ weeks gestational age (GA) achieve ductal constriction by day 4; however, in extremely premature infants, failure of constriction is common, affecting $70 \%$ in less than 28 weeks and $80 \%$ in $24-25$ weeks of gestation $[1,2]$.

The usual belief is that all patent ducts are pathologic is misconception. In some cases, the patent ductus may be an innocent and with little hemodynamic consequences, whereas in other situations, it may contribute to significant pathological changes and if delayed in

Manuscript received: $6^{\text {th }}$ February 2017

Reviewed: $14^{\text {th }}$ February 2017

Author Corrected: $20^{\text {th }}$ February 2017

Accepted for Publication: 28 ${ }^{\text {th }}$ February 2017 diagnosis and treatment can cause neonatal mortality [3]. As the Patent ductus is open, blood flows left-toright from the aorta into the pulmonary arteries.i As pulmonary vascular resistance decreases over the first few months after birth, the aortic blood flow that is moving into the pulmonary circulation correspondingly increases. This "ductal steal" results in excessive blood flow through the pulmonary circulation, predisposing to development of pulmonary congestion, pulmonary edema, and respiratory failure and resulting in compromised perfusion of vital organs, including bowel, kidney, and brain.

Prolonged ductal opening results in various adverse outcomes, including prolongation of assisted ventilation and higher rates of death, bronchopulmonary dysplasia (BPD), pulmonary hemorrhage, necrotizing enterocolitis, impaired renal function, intraventricular hemorrhage (IVH), periventricular leukomalacia, cerebral palsy The extent to which these adverse outcomes are attributable to the hemodynamic 
consequences of ductal patency. All this led to the hypothesis that interventions to close the ductus help in preventing the adverse effects.

A PDA is defined as hemodynamically significant when, in the presence of a left-to-right shunt, ultrasound criteria of hemodynamic impact (i.e. larger diameter of the DA associated with low velocity of blood through it, dilation of the left heart chambers, and diastolic steal in the systemic circulation) are also identified. The diagnosis of PDA must be prompt, ideally before the onset of clinical signs, especially in preterm babies in whom these signs can appear later $[4,5]$.

The goal of PDA treatment is to prevent short-term and long-term sequelae secondary to a high-volume ductal shunt. The intent is to prevent respiratory decompensation, heart failure, intraventricular hemorrhage (IVH), chronic lung disease (CLD), necrotizing enterocolitis (NEC), and death [6, 7]. Optimal management of the PDA in the premature infant is a controversial issue. Indomethacin was the first drug used, with the first study of its effectiveness in DA closure.

Response to indomethacin was defined as closed or non-significant PDA confirmed on echocardiogram after treatment with indomethacin. Dosage regimens used for a course of indomethacin were $0.2 \mathrm{mg} / \mathrm{kg} \times 3$ doses, $12 \mathrm{hrs}$ apart or $0.1 \mathrm{mg} / \mathrm{kg} \times 5$ to 6 doses, 24 hours apart. Later, ibuprofen was investigated in trials and it has increasingly been used in clinical practice. Ibubrufen is used at doses of $10 \mathrm{mg} / \mathrm{kg}$ orally or by Naso-gastric tube followed by $5 \mathrm{mg} / \mathrm{kg}$ later 24 hours apart for 2 days, a total cumulative dose of $20 \mathrm{mg} / \mathrm{kg}$. More recently, paracetamol has been introduced as an option in the treatment of PDA, showing very few side effects compared with other medications [8].

A second cycle of treatment is indicated if the first one fails to close the DA. Several studies comparing the safety and efficacy of indomethacin versus ibuprofen demonstrated equal efficacy in DA closure in preterm infants [9]. However, there are studies showing shorter duration of ventilatory support, fewer cases of Necrotizing enterocolitis (NEC) and a lower incidence of oliguria in the ibuprofen group $[10,11]$.

The purpose of the study is to see for the incidence and features of PDA in neonates admitted in the neonatal ICU and effectiveness of the Ibubrufen in treatment of hemodynamically significant Patent ductus.

\section{Materials and Methods}

A 5-year prospective observational study was done between January 2012 to December 2016 in the neonatal ICU of Vydehi institute of medical sciences. In this observational study, all the neonates suspected to have congenital heart disease in the NICU on the basis of history clinical examination, pulse oximetry, chest $\mathrm{X}$ ray were included in the study and confirmed by $2 \mathrm{D}$ echocardiography and colour Doppler study. A total of 2658 cases were admitted in neonatal ICU during this study period in which screening ECHO was done in 1974 cases. The same trained neonatologist performed clinical evaluation and ECHO within the first 24 hours of admission to the NICU. Screening ECHO was done on all the preterm babies, term babies with respiratory distress, murmur, features of CHF and all the babies requiring respiratory support like oxygen by hood or CPAP or ventilation [6]. PDA if detected was included in the study and treated if found hemodynamically significant, that was having ductal diameter more than $1.4 \mathrm{~mm}$, Pulse-wave Doppler of the descending aorta shows reversed end-diastolic flow, Pulse-wave Doppler in main pulmonary artery shows turbulent systolic and diastolic flow and large left-to-right shunt with a pulsatile flow pattern and the highest velocity at end systole. The babies were reviewed daily for 3 days, at 5 th day and at discharge. Standardized treatment protocol was given to all babies with hemodynamically significant PDA with Ibubrufen $10 \mathrm{mg} / \mathrm{kg}$ on day 1 and $5 \mathrm{mg} / \mathrm{kg}$ at 24 hours and at 48 hours. Size of the PDA is calculated in all the cases and considered small if $<1.4$ $\mathrm{mm}$, moderate sized PDA if between $1.4 \mathrm{~mm}$ to $2 \mathrm{~mm}$ and if more than $2 \mathrm{~mm}$ than considered large PDA. Response to treatment is considered if size of PDA becomes small, that is PDA less than $1.4 \mathrm{~mm}$ or completely closes by day 5 of starting the treatment or at discharge. If child expires or discharge against advice during course of treatment then the case is excluded from the study group. We conducted screening for SpO2 using Masimo Rad 7 pulse oximetry (Masimo Corporation, Irvine, CA, USA) and disposable probes. A trained neonatologist in neonatal ECHO using Hd11XE performed ECHO-Philips using mainly Cardiac probes 12-14.

Statistical analysis: Data were analyzed using Intercooled STATA version 10.0 for Windows. Categorical data were summarized using frequency distributions with $95 \%$ confidence intervals and continuous data were described using mean (standard deviation) when normally distributed or using medians and interquartile ranges otherwise. 
Original Research Article

\section{Results}

A total of 2658 cases were admitted in the NICU during the study period of which 1974 cases were screened for CHDs. Out of 1974 babies, Echo done showed 65 babies had PDA. Out of 65 cases with PDA 32 were hemodynamically significant.

Table-1: Demographic Distribution of PDA cases.

\begin{tabular}{|c|c|c|c|c|c|c|c|c|}
\hline & No. Of PDA & $\begin{array}{c}\text { Male } \\
/ \text { Female }\end{array}$ & $\begin{array}{c}\text { Term } \\
\text { /Preterm }\end{array}$ & $\begin{array}{c}\text { Small } \\
\text { PDA }\end{array}$ & $\begin{array}{c}\text { Medium } \\
\text { PDA }\end{array}$ & $\begin{array}{c}\text { Large } \\
\text { PDA }\end{array}$ & CPAP & Ventilation \\
\hline HDSA & 32 & $19 / 13$ & $3 / 29$ & 0 & 23 & 9 & 11 & 14 \\
\hline $\begin{array}{c}\text { Not } \\
\text { HDSA }\end{array}$ & 33 & $18 / 15$ & $9 / 24$ & 24 & 9 & 0 & 9 & 7 \\
\hline Total & 65 & $37 / 28$ & $12 / 53$ & 24 & 32 & 9 & 20 & 21 \\
\hline
\end{tabular}

Table 1 shows that out Of 32 HSDA cases, 29 were preterm requiring ventilation in 14 cases and CPAP in 11 cases. About 23 had medium sized PDA, which were hemodynamically significant ductus arteriosus (HSDA), and remaining 9 had large PDA. All the large sized PDAs were hemodynamically significant.

Table-2: Distribution of cases by birth weight and gestation.

\begin{tabular}{|c|c|c|c|c|c|}
\hline \multirow[t]{2}{*}{ Gestation age } & \multicolumn{4}{|c|}{$\begin{array}{l}\text { Weight (in gms) } \\
\text { No. of babies with PDA / Hemodynamically significant DA }\end{array}$} & \multirow[t]{2}{*}{ Total } \\
\hline & $>2500 \mathrm{gms}$ & 2500-1500gms & 1500-1000gms & $<1000$ gms & \\
\hline$>37$ weeks & $6 / 2$ & $3 / 1$ & - & - & $9 / 3$ \\
\hline 35-37 weeks & $3 / 1$ & $5 / 2$ & $2 / 0$ & - & $10 / 3$ \\
\hline 32-35 weeks & - & $7 / 3$ & $7 / 4$ & $1 / 1$ & $15 / 8$ \\
\hline 29-32 weeks & - & - & $9 / 3$ & $11 / 8$ & $20 / 11$ \\
\hline$<29$ weeks & - & - & $4 / 3$ & $7 / 4$ & $11 / 7$ \\
\hline & $9 / 3$ & $15 / 6$ & $22 / 10$ & $19 / 13$ & $65 / 32$ \\
\hline
\end{tabular}

Table 2 shows that out Of the 32 cases with HSDA on 3 were term neonates. 7 out of 11 neonates with gestation of $<29$ weeks had significant PDA.19 neonates with birth weight less $1000 \mathrm{gms}$ had PDA of which 13 were HSPD. All the large PDAs were hemodynamically significant.

Table-3: clinical signs of PDA.

\begin{tabular}{|c|c|}
\hline Symptoms & $\begin{array}{c}\text { No. (\%) } \\
(\mathbf{n}=\mathbf{6 5})\end{array}$ \\
\hline Systolic Murmur & $48(73 \%)$ \\
\hline Respiratory distress & $24(36 \%)$ \\
\hline CHF/hepatomegaly & $9(13 \%)$ \\
\hline Abnormal Chest X ray/cardiomegaly & $11(16 \%)$ \\
\hline Increased oxygen requirement & $6(9 \%)$ \\
\hline Recurrent Apnea & $3(4 \%)$ \\
\hline
\end{tabular}

Table 3 shows that Respiratory distress $(36 \%)$ is most common presenting symptom with increased oxygen requirement (24\%). Systolic murmur (73\%) is most common presenting sign requiring screening for any heart disease. 
Original Research Article

Table-4: Treatment response to hemodynamically significant PDA.

\begin{tabular}{|c|c|c|c|c|c|}
\hline Treatment & Number & Moderate/large PDA & By Day 3 & By day 5 & At discharge \\
\hline Response & 25 & $20 / 5$ & $6(24 \%)$ & $12(48 \%)$ & $7(28 \%)$ \\
\hline No response & 7 & $3 / 4$ & - & - & - \\
\hline
\end{tabular}

Table 4 shows that Around 25 out of 32 cases had responded to treatment with Ibubrufen. Ductus were closed in 5 out of 9 large PDAs. About $48 \%$ of the PDAs were closed by $5^{\text {th }}$ day of starting the treatment.

\section{Discussion}

The study shows that patent ductus arteriosus is a commonest Acyanotic congenital heart disease in the neonates admitted in the NICU. The incidence was 2.4 cases per 1000 live births comparable to study done by shenoy et.al in which it were 2.8 cases per 1000 live births. There was no significant difference between male and female PDA in both significant and non significant cases of PDA.Of the 65 cases only 32 (49\%) had hemodynamically significant ductus arteriosus. The study shows the incidence of PDA in 1500 to $2500 \mathrm{gm}$, $1000-1500$ gms and $<1000$ gms are respectively $32 \%$, $29 \%$ and $49 \%$ of the total cases comparable to $16 \%$, $36 \%$ and $46 \%$ in study by Ellison et.al in USA [12].

Around $71 \%$ of the HSPA had requirement of ventilation in the form of CPAP or ventilator.PDA being a complication of Hyaline membrane disease is manifested by increased requirement of oxygen in the form of ventilation or CPAP. This was seen in 6 babies in our study, which on treatment were able to wean of from oxygen requirement. Recurrent Apnea was seen in 3 neonates and was hemodynamically significant and required treatment.

Four babies with PDA and sepsis with pneumonia died during stay in the NICU were not included in the study. All the babies with PDA were restricted with fluids (maximum of $120-140 \mathrm{ml} / \mathrm{kg} /$ day).

In as study by popet et al, 7\% underwent surgical ligation during the study period which in effect leaves $93 \%$ of the infants managed non-surgically [13]. This is comparable with our study where 7 out of 65 cases of PDA that is around $10 \%$ underwent surgical ligation.

All HSPD babies were treated with Ibubrufen and successful closure was seen in $78 \%$ comparable to study done by shenoy et.al at $75 \%$. Around $28 \%$ of the HSPA which did not respond to Ibubrufen underwent surgical closure. Several studies have shown that single dosage regimen of Ibubrufen had lower success rates compared to that of step wise escalation of the drug has success rates of more than $90 \%$ but chances of increased side effects were noted [13]. Non response to Ibubrufen is more in pre terms than terms due to multifactorial causes. No side effects were observed because of the drug. Neonates with PDA significantly better responded to Ibubrufen with $\mathrm{p}$ value $<0.005$.

\section{Conclusion}

The study shows PDA is a commonest Acyanotic CHD in NICU and early administration of the drug is helpful in closure, avoiding surgical correction later and also mortality. Studies need to be done to study the role of multiple courses of Ibubrufen is required for closure to avoid surgical correction and usage of even in PDA even if not significant hemodynamically.

Funding: Nil, Conflict of interest: None initiated, Perission from IRB: Yes

\section{References}

1. Ufuk Kursat Korkmaz, Esra Akyuz Ozkan, Ayse Neslin Akkoca, Ebru Sivaslioglu. Incidence and Clinical Characteristics of Congenital Heart Disease among Neonates in Neonatal Intensive Care Unit. American Journal of Health Research. Vol. 3, No. 3, 2015, pp. 161-165. doi: 10. 11648/j. ajhr. 20150 303.19

2. Koch J, Hensley G, Roy L, Brown S, Ramaciotti C, Rosenfeld CR. Prevalence of spontaneous closure of the ductus arteriosus in neonates at a birth weight of 1000 grams or less. Pediatrics. 2006 Apr;117(4):1113-21.

3. Hussain S, Sabir MU, Afzal M, Asghar I. Incidence of congenital heart disease among neonates in a neonatal unit of a tertiary care hospital. J Pak Med Assoc. 2014 Feb;64(2):175-8.

4. Heymann MA, Rudolph AM, Silverman NH. Closure of the ductus arteriosus in premature infants by inhibition of prostaglandin synthesis. N Engl J Med. 1976 Sep 2;295(10):530-3. 
5. Wren C, Richmond S, Donaldson L. Presentation of congenital heart disease in infancy: implications for routine examination. Arch Dis Child Fetal Neonatal Ed. 1999 Jan;80(1):F49-53.

6. Fatema NN, Chowdhury RB, Chowdhury L. Incidence of congenital heart disease among hospital live births in a tertiary hospital of Bangladesh. Cadiovasc J 2008 Mar; 1: 14-20.

7. Hoffman JI, Kaplan S. The incidence of congenital heart disease. J Am Coll Cardiol. 2002 Jun 19;39 (12): 1890-900.

8. Yang XY, Li XF, Lü XD, Liu YL. Incidence of congenital heart disease in Beijing, China. Chin Med J (Engl). 2009 May 20;122(10):1128-32.

9. Gupta A, Abqari S, Shahab T, Rabbani MU, Ali SM, Firdaus U. Profile and Clinical Spectrum of Congenital Heart Defects in NICU. Int Arch BioMed Clin Res.
2016 April;2(2):25-27.

10. Skelton R, Evans N, Smythe J. A blinded comparison of clinical and echocardiographic evaluation of the preterm infant for patent ductus arteriosus. J Paediatr Child Health. 1994; 30:406411.

11. Hirsimaki H, Kero P, Wanne O (1990) Doppler ultrasound and clinical evaluation in detection and grading of patent ductus arteriosus in neonates. Crit Care Med 18:490-493.

12. Clyman RI, Chorne N. Patent ductus arteriosus: evidence for and against treatment. J Pediatr. 2007 Mar; 150 (3):216- 19.

13. Popat H, Kapoor v, Travadi J.Patent Ductus Arteriosus in Infants <29 Weeks Gestation - Outcomes and Factors Affecting Closure. Indian Pediatrics.2012 Aug; 49 (8):615-20.

\section{How to cite this article?}

Dinakara P, Bharat Reddy D, Rajeshwari S, Utkarsh J. Patent ductus arteriosus - clinical profile and outcome after treatment in a tertiary care hospital. J PediatrRes. 2017;4(02):167-171.doi:10.17511/ijpr.2017.i02.13. 